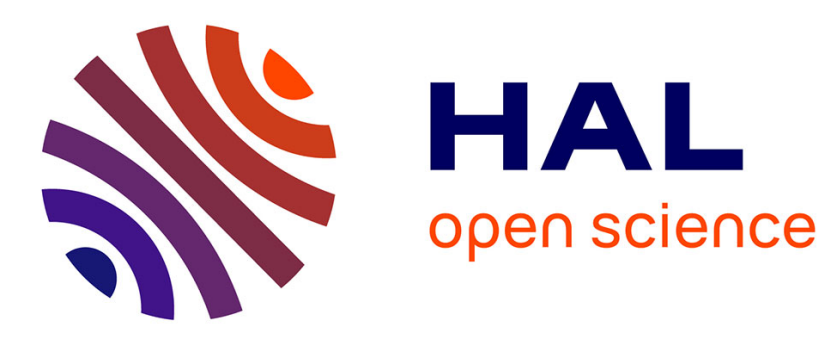

\title{
Égalité négociée, égalité standardisée ?
}

Marion Charpenel, Hélène Demilly, Sophie Pochic

\section{To cite this version:}

Marion Charpenel, Hélène Demilly, Sophie Pochic. Égalité négociée, égalité standardisée ?. Travail, genre et sociétés, 2017, $\mathrm{n}^{\circ}$ 37, pp.143-147. 10.3917/tgs.037.0143 . halshs-02079907

\section{HAL Id: halshs-02079907 https://shs.hal.science/halshs-02079907}

Submitted on 13 Oct 2021

HAL is a multi-disciplinary open access archive for the deposit and dissemination of scientific research documents, whether they are published or not. The documents may come from teaching and research institutions in France or abroad, or from public or private research centers.
L'archive ouverte pluridisciplinaire HAL, est destinée au dépôt et à la diffusion de documents scientifiques de niveau recherche, publiés ou non, émanant des établissements d'enseignement et de recherche français ou étrangers, des laboratoires publics ou privés. 


\section{Marion Charpenel, Hélène Demilly et Sophie Pochic}

\section{Égalité négociée, égalité standardisée?}

En France, l'appareil législatif qui encadre la négociation collective de l'égalité professionnelle n'a cessé d'évoluer depuis la loi Génisson de 2001, qui impose aux entreprises de se doter d'un accord triennal ou, à défaut, d'un plan annuel sur cette question. En quinze ans, de nombreux textes réglementaires sont venus renforcer cette obligation et modifier les procédures, le périmètre, ainsi que le calendrier des négociations. L'instauration d'une pénalité financière visant les entreprises contrevenantes a indéniablement accru la production de textes depuis 2012, bien que des contrastes persistent selon la taille des entreprises. Ainsi, en février 2016, $84 \%$ des entreprises de plus de 1000 salariés satisfont à cette exigence, contre seulement $34 \%$ des entreprises de 50 à 299 salariés. Des secteurs jusque-là peu concernés par cette négociation - tels que la construction, le commerce, et la santé - l'intègrent progressivement à leur agenda social. Mais cette initiative laissée au secteur privé ne risque-t-elle pas d'aboutir à des " coquilles vides » plutôt qu'à de réelles politiques d'égalité ? Les réflexions présentées ici s'appuient sur l'étude ${ }^{1}$ de cent-quatre-vingt-six textes signés entre 2014 et 2015 (quatre-vingt-dix-huit accords négociés et quatrevingt-huit plans unilatéraux, dont sept associés à des procès-verbaux de désaccords). L'échantillon de documents respecte globalement, pour chaque secteur étudié ${ }^{2}$, la proportion d'établissements ayant enregistré un texte auprès de l'administration du travail. Par ailleurs, il fait varier le taux de féminisation des entreprises, leur taille, le profil de qualification de leur main-d'œuvre, leur inscription éventuelle dans un groupe ainsi que leurs pratiques de négociation.

L'évolution fréquente des dispositions légales crée indéniablement de l'incertitude et de la complexité face auxquelles directions et représentants du personnel sont inégalement armés : selon les ressources dont ils disposent, les secteurs dans lesquels ils s'insèrent, ou encore la santé économique de leur entreprise, leur implication sur ce thème, leurs motivations et leurs marges de manœuvre sont variables. Pourtant, en regard d'autres études ayant choisi le même protocole d'enquête ${ }^{3}$, le premier constat marquant est celui de la relative standardisation des textes tant sur la forme que sur le contenu. Au-delà d'une perspective évaluative des dispositifs d'action publique qui laissent à la négociation

\footnotetext{
${ }^{1}$ Étude réalisée pour la Dares, coordonnée par Sophie Pochic, avec Delphine Brochard, Vincent-Arnaud Chappe, Marion Charpenel, Hélène Demilly, Susan Milner et Marion Rabier.

${ }^{2}$ Les dix secteurs étudiés, par ordre croissant de taux de féminisation, sont la construction, la métallurgie, l'électricité et le gaz, le transport, l'informatique et l'ingénierie, l'industrie agroalimentaire, les études et le conseil, la banque et la finances, le commerce et la santé (humaine) privée.

${ }^{3}$ Parmi les nombreuses études, on citera Jacqueline Laufer et Rachel Silvera [2006] ; Marion Rabier [2008] ; Anne Bucher et al. [2015].
} 
${ }^{4}$ Forme d'aide à la décision fondée sur des preuves de type scientifique, notamment quantitatives [Laurent et al., 2009].

${ }^{5}$ Un « rapport écrit sur la situation comparée des conditions générales d'emploi et de formation des femmes et des hommes dans l'entreprise ", Art. L. 2323-57 du Code du travail.

${ }^{6}$ Ce qui s'explique notamment par le risque juridique à reconnaître l'existence d'un écart de salaire entre les femmes et les hommes, dans l'éventualité d'un procès pour discrimination [Silvera, 2014]. d'entreprise le pouvoir de dire le droit, cette observation a priori surprenante pose des questions en termes de sociologie des relations professionnelles. Comment expliquer qu'une législation qui vise à favoriser la prise en compte des situations locales aboutisse à des productions homogènes? L'analyse des arguments et dispositifs présents dans ces textes permet de dégager trois éléments de réponse : tout d'abord, des difficultés pour les auteurs des textes à objectiver la structure des inégalités sexuées dans l'entreprise et donc à adapter les mesures à la situation locale ; ensuite, la faiblesse générale des budgets alloués à l'égalité, qui contraint le choix des moyens d'action et conduit à leur standardisation ; enfin, une approche de l'égalité souvent réduite à la question de la mixité, ce qui a pour effet de concentrer l'attention des partenaires sociaux sur un nombre limité de problématiques et sur certaines catégories de salarié·e·s.

\section{Diagnostiquer les inégalités : les limites de 1'evidence-based policy ${ }^{4}$}

Dès la création du rapport de situation comparée ${ }^{5}$ en 1983, les pouvoirs publics ont adossé les politiques d'égalité professionnelle à une objectivation quantifiée des inégalités. Les mobilisations contre la suppression de ce rapport, prévue par la loi Rebsamen de 2015, ont réaffirmé l'importance du diagnostic chiffré pour orienter les actions négociées et suivre leur mise en œuvre. Cependant, près de $40 \%$ des textes de notre corpus ne mobilisent aucun chiffre. Les données fournies sont souvent incomplètes, en particulier concernant la rémunération ${ }^{6}$ : par exemple, les tableaux intégrés aux textes fournissent en général la rémunération moyenne sexuée par catégorie socioprofessionnelle, mais ne donnent pas la rémunération moyenne femmes-hommes dans l'établissement. Cela empêche de construire une analyse dynamique et systémique reliant ces écarts au plafond de verre ou à la sous-valorisation des emplois à dominante féminine. Souvent, l'étude est aussi restreinte à l'analyse des salaires de base, sans intégrer les primes et bonus pourtant très discriminants. Par ailleurs, des entreprises se contentent de reproduire des chiffres sans les commenter, ce qui entretient une ambiguité sur le fait que les écarts statistiques sexués ont bien été interprétés comme des inégalités à justifier, et a fortiori comme des discriminations à corriger.

Dans le domaine de l'égalité professionnelle, ce constat dessine les limites de l'evidence-based policy, qui voudrait que la production de preuves objectives suffise à déterminer les contours d'actions appropriées. En effet, les chiffres ne parlent pas d'eux-mêmes, et leur production comme leur interprétation sont déterminées par les cadres de pensée des négociateurs. Un procès-verbal de désaccord entre les organisations syndicales et la direction d'un groupe 
international de conseil et d'ingénierie montre que les conflits peuvent porter sur le diagnostic lui-même : la direction se satisfait de «l'absence d'écart significatif » de rémunération, tandis que les élus syndicaux constatent " un écart systématique en défaveur des femmes ». Ces discussions illustrent la difficulté des négociateurs à s'accorder sur la structure des inégalités sexuées dans leur organisation. Ces défaillances dans la production et l'analyse des chiffres, qu'elles résultent d'un manque de formation des acteurs ou d'une divergence de points de vue [Cristofalo, 2014], pourraient constituer un premier facteur de standardisation des textes, favorisant des mesures déconnectées de la situation locale de l'entreprise.

\section{Du droit, du management : le kit de l'égalité «bon marché »}

La loi oblige les entreprises à évaluer le coût des mesures prévues dans les plans d'action ou les accords ${ }^{7}$. Pourtant, seuls cinquante-sept textes sur cent-quatre-vingt-six sont conformes à cette obligation légale. Par ailleurs, les quelques budgets présents laissent apparaître une tendance à mettre en œuvre l'égalité à moindre coût. Six entreprises admettent même explicitement que "les mesures n'entraîneront aucun surcoût » ou qu'elles sont estimées à "zéro euro ». Corollaire de ce souci de limiter les dépenses, de nombreuses " mesures » présentées comme des actions ne sont en fait que des déclarations de principes ou consistent en des rappels du droit, énonçant par exemple l'interdiction de «toute question pouvant se révéler comme discriminante lors des entretiens de recrutement $»$.

Par ailleurs, les mesures collectives, plus coûteuses (enveloppes de rattrapage, révision des classifications ou transformation des processus organisationnels), sont les moins fréquentes. Les textes tendent plutôt à privilégier une approche " au cas par cas", qui repose principalement sur l'engagement des managers et des services de ressources humaines. En témoigne l'usage de l'entretien individuel comme mode d'action privilégié, qu'il s'agisse d'aménager l'emploi du temps, d'accorder une promotion ou d'examiner des réclamations salariales. L'égalité est donc généralement incorporée dans les dispositifs de gestion individualisée du personnel, sans analyser les éventuels biais discriminants ou sexistes des outils de management par les compétences et les objectifs. Les accords et plans d'action apparaissent alors comme des dispositifs hybrides, incorporant des éléments rhétoriques et des mesures issus des registres juridique et gestionnaire.

\section{Le prisme de la mixité, une égalité pour les mieux doté·e·s}

La lecture des accords et plans d'action donne à voir une certaine confusion entre " mixité » et " parité », qui semblent 
prendre le pas sur la notion d'égalité, quel que soit le taux de féminisation du secteur. Ainsi, les mesures envisagées en matière d'embauche, de formation ou encore de conditions de travail servent un objectif de "représentation équilibrée » des femmes et des hommes, ce qui correspond à une catégorie mobilisée par les pouvoirs publics dans les années récentes, à l'instar de la loi Copé-Zimmermann de 2011.

Ce prisme a deux grandes conséquences sur le choix des publics cibles. Tout d'abord, cette " grammaire paritaire» [Bereni et Revillard, 2007] conduit à une focalisation, en termes d'actions positives, sur l'accès des femmes aux postes à responsabilités. Ainsi, de nombreuses mesures concernent prioritairement les femmes cadres, à l'instar des programmes de coaching ou de mentoring prévus pour celles qui accèdent à une position managériale. Reflet d'une tendance à l' « égalité élitiste » [Jacquemart, Le Mancq et Pochic, 2016], ces textes négligent les problématiques propres aux ouvrières et employées tels que les emplois précaires ou les horaires atypiques.

Le prisme de la mixité amène aussi certaines entreprises à prendre, au nom de l'égalité, des mesures centrées sur les hommes. Si, dans le domaine de l'articulation entre travail et famille, il est essentiel de promouvoir un meilleur partage des tâches, cette logique de symétrie conduit parfois à nier la domination masculine. Certaines actions positives en faveur des hommes, dans les domaines de l'embauche et de la promotion, ignorent ainsi le phénomène de l'« escalator de verre » [Williams, 2013] selon lequel les hommes connaissent structurellement des progressions de carrière accélérées dans les métiers féminisés.

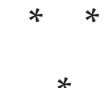

L'analyse de notre corpus de textes permet donc de conclure à une forme de standardisation de l'égalité, que ce soit entre secteurs d'activité (aux taux de féminisation contrastés) ou entre types de textes. On peut ainsi s'étonner de la faible différence entre les accords - signés par au moins une organisation syndicale - et les plans d'action élaborés unilatéralement par la direction. Des monographies d'entreprise permettront d'explorer ce paradoxe en réinscrivant ces textes dans leur contexte de production, et notamment dans les rapports de coopération ou de conflit entre les acteurs (directions, structures syndicales, experts et consultants, réseaux de femmes cadres, délégués du personnel).

\section{RÉFÉRENCES BIBLIOGRAPHIQUES}

BERENI Laure et REVILLARD Anne, 2007, « Des quotas à la parité : “féminisme d'État" et représentation politique (1974-2007) », Genèses, n 67, pp. 5-23. 
Bucher Anne, Forte Michèle, Garat Tiphaine, Moizard Nicolas, Terraz Isabelle et TOURNADRE Fabienne, 2015, Négocier l'égalité professionnelle entre les femmes et les hommes : Avancées, résistances et conditions de développement, Rapport pour la Direccte Alsace, avril.

CRistofalo Paola, 2014, «Négocier l'égalité professionnelle: de quelques obstacles à la prise en charge syndicale de la thématique », Nouvelle Revue de Psychosociologie, vol. 18, n², pp. 133-146.

GARNER Hélène et ReCoules Magali, 2014, Égalité, diversité, discriminations. Étude de 80 accords d'entreprise sur la diversité, Document DARES, $\mathrm{n}^{\circ} 182$.

Jacquemart Alban, Le Mance Fanny et Pochic Sophie, 2016, «Femmes hautes fonctionnaires en France, l'avènement d'une égalité élitiste? », Travail, genre et sociétés, $\mathrm{n}^{\circ} 35$, pp. 27-45.

LAufer Jacqueline et Silvera Rachel, 2006, «L'égalité des femmes et des hommes en entreprise. De nouvelles avancées dans la négociation? », Revue de l'OFCE, $\mathrm{n}^{\circ}$ 97, pp. 245-271.

Laurent Catherine, Baudry Jacques, Berriet-Solliec Marielle, KIrsch Marc, Perraud Daniel, Tinel Bruno, Trouvé Aurélie, Allsopp Nicky, Bonnafous Patrick, Burel Françoise, Carneiro Maria José, Giraud Christophe, Labarthe Pierre, Matose Frank et Ricroch Agnès, 2009, "Pourquoi s'intéresser à la notion d'“evidence-based policy" ? ", Revue Tiers Monde, n² 200, p. 853-873.

RABIER Marion, 2008, «Analyse du contenu des accords d'entreprise portant sur l'égalité professionnelle entre les femmes et les hommes signés depuis la loi du 23 mars 2006 ", Dares-DGT, La négociation collective en 2008.

SILVERA Rachel, 2014, Un quart en moins. Des femmes se battent pour en finir avec les inégalités de salaires, Paris, La Découverte.

Williams Christiane, 2013, « The Glass Escalator, Revisited. Gender Inequality in Neoliberal Times », Gender E Society, vol. 27, n 5, pp. 609-629. 